\title{
Coronary computed tomography angiography-adapted Leaman score as a tool to noninvasively quantify total coronary atherosclerotic burden
}

\author{
Pedro de Araújo Gonçalves - Hector M. Garcia-Garcia • Helder Dores • \\ Maria Salomé Carvalho · Pedro Jerónimo Sousa - Hugo Marques • \\ Antonio Ferreira $\cdot$ Nuno Cardim $\cdot$ Rui Campante Teles $\cdot$ Luís Raposo \\ Henrique Mesquita Gabriel - Manuel Sousa Almeida • Ana Aleixo • \\ Miguel Mota Carmo • Francisco Pereira Machado • Miguel Mendes
}

Received: 19 April 2013/Accepted: 24 April 2013

(C) Springer Science+Business Media Dordrecht 2013

\begin{abstract}
To describe a coronary computed tomography angiography (CCTA)-adapted Leaman score (CT-LeSc) as a tool to quantify total coronary atherosclerotic burden with information regarding localization, type of plaque and degree of stenosis and to identify clinical predictors of a high coronary atherosclerotic burden as assessed by the CT-LeSc. Single center prospective registry including a total of 772 consecutive patients undergoing CCTA (Dualsource CT) from April 2011 to March 2012. For the purpose of this study, 581 stable patients referred for suspected coronary artery disease (CAD) without previous myocardial infarction or revascularization procedures were included. Pre-test CAD probability was determined using both the Diamond-Forrester extended CAD consortium method
\end{abstract}

P. de Araújo Gonçalves $(\bowtie) \cdot H$. Dores ·

M. S. Carvalho · P. Jerónimo Sousa · A. Ferreira .

R. Campante Teles - L. Raposo - H. Mesquita Gabriel ·

M. Sousa Almeida - A. Aleixo - M. Mendes

Cardiology Department, Centro Hospitalar Lisboa Ocidental,

Lisbon, Portugal

e-mail: paraujogoncalves@yahoo.co.uk

P. de Araújo Gonçalves · H. Marques · A. Ferreira ·

N. Cardim - F. Pereira Machado

Hospital da Luz, Lisbon, Portugal

P. de Araújo Gonçalves · A. Aleixo · M. Mota Carmo CEDOC, Chronic Diseases Research Center, FCM-NOVA, Lisbon, Portugal

H. M. Garcia-Garcia

Thoraxcenter, Erasmus MC, Rotterdam, The Netherlands
(DF-CAD consortium model) and the Morise score. Cardiovascular risk was assessed with the HeartScore. The cutoff for the 3rd tercile (CT-LeSc $\geq 8.3$ ) was used to define a population with a high coronary atherosclerotic burden. The median CT-LeSc in this population $(\mathrm{n}=581,8,136$ coronary segments evaluated; mean age $57.6 \pm 11.1$; $55.8 \%$ males; $14.6 \%$ with diabetes) was 2.2 (IQR 0-6.8). In patients with CAD $(\mathrm{n}=341)$, the median CT-LeSc was 5.8 (IQR 3.2-9.6). Among patients with nonobstructive $\mathrm{CAD}$, most were classified in the lowest terciles (T1, $43.0 \%$; T2, $36.1 \%$ ), but $20.9 \%$ were in the highest tercile (T3). The majority of the patients with obstructive CAD were classified in T3 $(78.2 \%)$, but $21.8 \%$ had a CT-LeSc in lower terciles (T1 or T2). The independent predictors of a high CT-LeSc were: Male sex (OR 1.73; $95 \%$ CI 1.04-2.90) diabetes (OR 2.91; $95 \%$ CI 1.61-5.23), hypertension (OR 2.54; $95 \%$ CI 1.40-4.63), Morise score $\geq 16$ (OR 1.97; $95 \%$ CI 1.06-3.67) and HeartScore $\geq 5$ (OR 2.42; $95 \%$ CI 1.41-4.14). We described a cardiac CT adapted Leaman score as a tool to quantify total (obstructive and nonobstructive) coronary atherosclerotic burden, reflecting the comprehensive information about localization, degree of stenosis and type of plaque provided by CCTA. Male sex, hypertension, diabetes, a HeartScore $\geq 5 \%$ and a Morise score $\geq 16$ were associated with a high coronary atherosclerotic burden, as assessed by the CTLeSc. About one fifth of the patients with nonobstructive CAD had a CT-LeSc in the highest tercile, and this could potentially lead to a reclassification of the risk profile of this subset of patients identified by CCTA, once the prognostic value of the CT-LeSc is validated.

Keywords CCTA - Coronary artery disease Atherosclerotic burden · Risk scores 


\section{Introduction}

Coronary atherosclerosis is the leading cause of mortality and it is expected to remain the most important disease in the upcoming years [1]. Frequently, the first manifestation of coronary disease is an acute coronary syndrome (ACS), and many patients were previously asymptomatic [2]. An early detection of coronary disease is of utmost relevance and a non-invasive diagnostic test is desirable.

In the recent years, coronary computed tomography angiography (CCTA) has become widely available and adopted. The main reason for this is the high predictive accuracy of detection of obstructive coronary artery disease (CAD) compared to conventional invasive coronary angiography [3, 4]. In addition, CCTA allows also the identification of nonobstructive $\mathrm{CAD}$ and in this way it can provide a noninvasive quantification of the total coronary atherosclerotic burden. Since the percentage of patients with nonobstructive CAD is very high, there is a need for tools to stratify cardiovascular risk by the degree of plaque burden [5]. The information regarding the localization, severity and composition of coronary plaques identified with CCTA can be collected in scores to reflect the total coronary plaque burden, and some have been already developed and validated [6].

Conventional cardiovascular (CV) risk factors relate to the risk of subsequent $\mathrm{CV}$ events and they can be combined in tools as it has been done in the Heart Score [7]. Notwithstanding these observations, accurate prediction of major coronary events on the individual patient level, as opposed to population based studies, remains challenging.

Therefore the aim of this study is two folded: (1) To describe a CCTA-adapted Leaman score (CT-LeSc) as a tool to quantify total coronary atherosclerotic burden including information regarding localization, type of plaque and degree of stenosis and; (2) To identify clinical predictors of a high coronary atherosclerotic burden as assessed by CT-LeSc in a population of stable patients referred for CCTA for suspected CAD.

\section{Methods}

Population

Single center prospective registry including a total of 772 consecutive patients undergoing CCTA (with Dual source CT) from April 2011 to March 2012. Patients were excluded if: (1) previous myocardial infarction and/or revascularization procedures $(\mathrm{n}=70)$; (2) referred for Cardiac CT for other indications than the evaluation of possible CAD (cardiac CT for atrial fibrillation ablation or transcatheter aortic valvular implantation procedures; $\mathrm{n}=88$ ); (3) referred for suspected ACS $(\mathrm{n}=24)$; (4) with atrial fibrillation or other significant arrhythmias during scan acquisition that compromised image quality $(n=9)$. This resulted in a $24.7 \%$ of the total population being excluded.

For the purpose of this study, 581 stable patients referred for suspected CAD were included in the context of: (1) Previous equivocal or inconclusive stress tests or discordant with the clinical evaluation $(\mathrm{n}=417 ; 71.8 \%)$; (2) Cardiac CT as 1st line investigation of possible CAD $(\mathrm{n}=136 ; 23.4 \%)$; (3) Preoperative CAD assessment prior to noncoronary valvular or aortic surgery $(\mathrm{n}=17 ; 2.9 \%)$; (4) Evaluation of possible CAD in cardiomyopathies (DCM or HCM; $\mathrm{n}=11 ; 1.9 \%$; Fig. 1: Patient selection and study design).

The study was approved by the local ethics committee and all patients gave a written informed consent.

A detailed medical history with a risk factors questionnaire was obtained from the patients to assess for the presence of: (1) Diabetes mellitus (defined as a fasting glucose level of $\geq 7 \mathrm{mmol} / \mathrm{l}$ or the need for insulin or oral hypoglycemic agents) [8]; (2) Dyslipidemia (defined as a total cholesterol level $\geq 5 \mathrm{mmol} / \mathrm{l}$ or treatment with lipid-lowering drugs) [9]; (3) Hypertension (defined as blood pressure $\geq 140 / 90 \mathrm{~mm} \mathrm{Hg}$ or the use of antihypertensive medication) [10]; (4) Obesity (body mass index $\geq 30 \mathrm{~kg} / \mathrm{m}^{2}$ ); (5) positive family history of premature CAD (defined as the presence of CAD in first-degree relatives younger than 55 [male] or 65 [female] years of age) [11]; (6) smoking (defined as previous [less $<1$ year] or current smoker.

Pre-test probability of CAD was determined using both the Diamond and Forrester extended CAD consortium method (DF-CAD consortium model) [12] and the Morise score [13]. The cardiovascular risk was assessed with the HeartScore [7]. As the CAD probability and CV risk of our population was shifted to lower probability and risk, the cut-offs used were: (1) for DF-CAD consortium model categories $\geq 30-70$ and $\geq 70 \%$ were gathered in a Intermediate to High ( $\geq 30 \%)$ probability group.

For the Morise, the population was divided in terciles, and for the HeartScore the established high risk cut-off of $\geq 5 \%$ was used.

Scan protocol and image reconstruction

All scans were performed with a dual-source scanner (Somatom Definition, Siemens Medical, Germany), with the patient in dorsal decubitus and in deep inspiration breath-hold. Sublingual nitroglycerin was administered to all patients except when contraindicated and intravenous metoprolol $(5 \mathrm{mg}$, with a titration dose up to $20 \mathrm{mg}$ ) was administered in patients with heart rate $>65$ beats $/ \mathrm{min}$.

During the scan acquisition, a bolus of iodinated contrast (Visipaque, GE Healthcare, USA) was injected at a 
Fig. 1 Patient selection and study design. $C A D$ coronary artery disease, $T A V I$ transcatheter aortic valve implantation, $a F i b$ atrial fibrillation, $M I$ myocardial infarction, $C A B G$ coronary artery bypass grafting, $P C I$ percutaneous coronary intervention, $A C S$ acute coronary syndromes

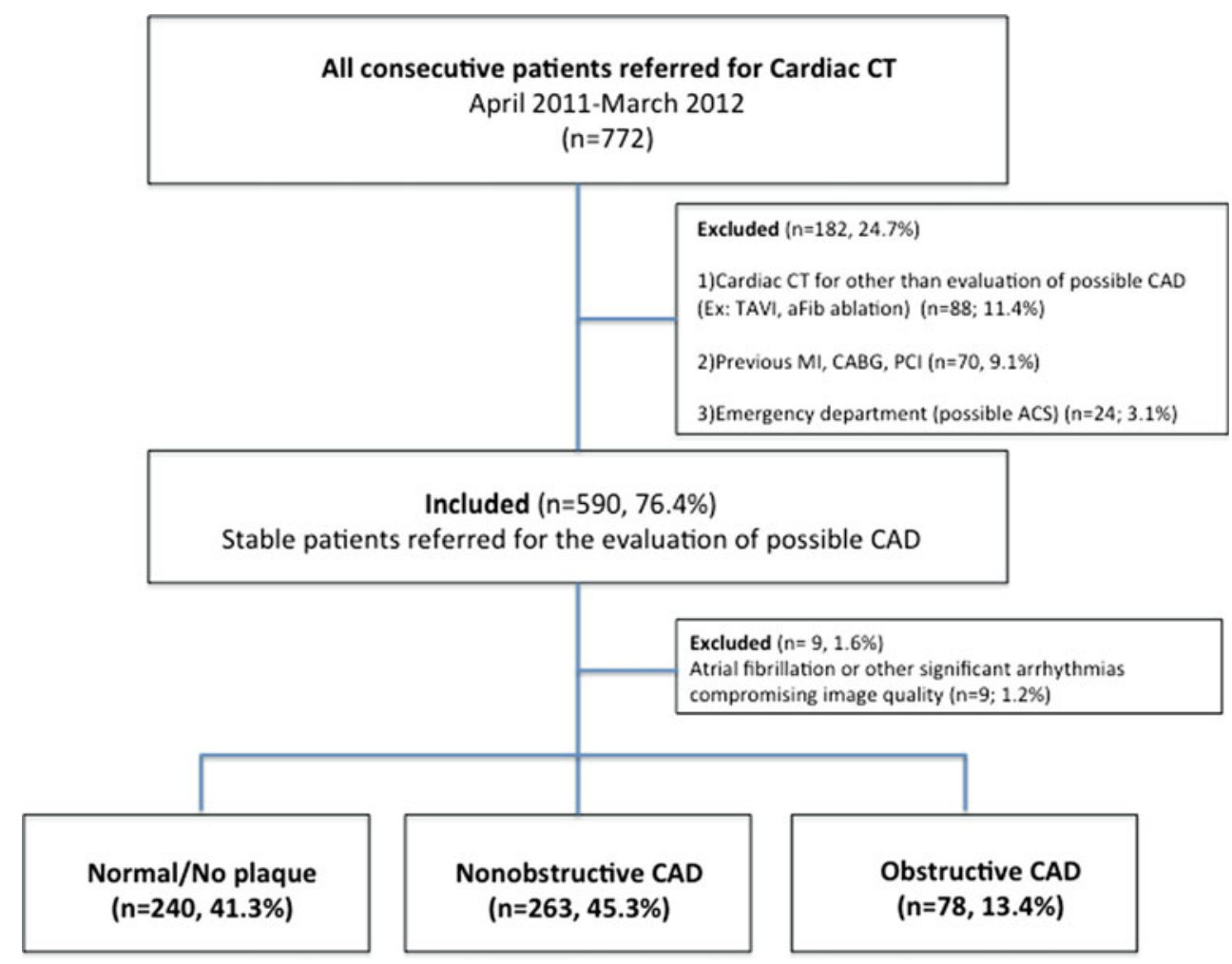

$6 \mathrm{ml} / \mathrm{s}$ infusion rate, followed by a $50-\mathrm{ml}$ saline flush. The dose of contrast was calculated according to the following formula: (acquisition time $+6 \mathrm{~s}$ delay) $\times$ flow $(6 \mathrm{ml} / \mathrm{s})$. Contrast timing was performed to optimize uniform contrast enhancement of the coronary arteries.

Dose reduction strategies - including electrocardiogramgated tube current modulation, reduced tube voltage, and prospective axial triggering - were used whenever feasible. Mean estimated radiation dose was $4.6 \pm 3.7 \mathrm{mSv}$, contrast dose was $98.9 \pm 14.4 \mathrm{ml}$ and heart rate was $65.6 \pm 10.6 \mathrm{bpm}$.

Transaxial images were reconstructed with a temporal resolution of $83 \mathrm{~ms}$ and slice thickness of $0.75 \mathrm{~mm}$ with $0.4 \mathrm{~mm}$ increments.

Post-processing was carried out using Circulation ${ }^{\circledR}$ software, with multiplanar reconstructions, maximum intensity projection and volume rendering technique.

Coronary artery analysis

All scans were analyzed in the same session by both a cardiologist and a radiologist with Level III-equivalent experience. The Society of Cardiovascular Computed Tomography recommended classification was used regarding segmentation (16 segments), stenosis severity $(<25$,
25-49, 50-69, 70-99, $100 \%$ ) and plaque composition (calcified, non calcified, mixed plaque) [14].

In each coronary artery segment, coronary atherosclerosis was defined as a tissue structure $>1 \mathrm{~mm}^{2}$ that existed either within the coronary artery lumen or adjacent to the coronary artery lumen that could be discriminated from surrounding pericardial tissue, epicardial fat, or the vessel lumen itself [6]. Coronary atherosclerotic lesions were quantified for stenosis by visual estimation. Percent obstruction of coronary artery lumen was based on a comparison of the luminal diameter of the segment exhibiting obstruction to the luminal diameter of the most normal-appearing site immediately proximal to the plaque.

\section{CCTA adapted Leaman score (CT-LeSc)}

For the CT adaptation of the LeSc, we used three sets of weighting factors, all noninvasively provided by CCTA: (1) localization of the coronary plaques as originally described [15]. In this study, a modification was made to account for balanced dominance. In cases of balanced dominance, not taken in account in the original Leaman or in the Syntax scores, we assumed an intermediate value between right and left dominance which changed the values for the posterior descending and the proximal, mid and 
distal RCA segments as well as for the left main and proximal and distal segments of the circumflex; (2) type of plaque (i.e. noncalcified, calcified or mixed plaques). To take in account the cardiac $\mathrm{CT}$ added information related to plaque composition, an additional weighting factor of 1.5 was added to predominantly noncalcified of mixed plaques and a factor of 1 to predominantly calcified plaques, reflecting the assumption of less plaque vulnerability of the later ones [16, 17]; (3) degree of stenosis $(<50 \geq \%$ stenosis). In the presence of obstructive CAD ( $\geq 50 \%$ stenosis), the score in each segment was multiplied by 1 and for nonobstructive CAD it was multiplied by a factor of 0.615. This factor reflects the relative proportion in the published hazard ratios for mortality in the large CONFIRM registry [5] for obstructive versus nonobstructive CAD (2.6 vs 1.6 respectively) and it was assumed to reflect the relative prognostic impact of nonobstructive CAD (Table 1).

Table 1 CT-adapted Leaman Score (CT-LeSc) weighting factors

\begin{tabular}{|c|c|c|c|}
\hline Segment & $\begin{array}{l}\text { Right } \\
\text { dominance }\end{array}$ & $\begin{array}{l}\text { Left } \\
\text { dominance }\end{array}$ & Balanced \\
\hline \multicolumn{4}{|l|}{ Coronary segments } \\
\hline RCA proximal & 1 & 0 & 0.5 \\
\hline RCA mid & 1 & 0 & 0.5 \\
\hline RCA distal & 1 & 0 & 0.5 \\
\hline PDA & 1 & na & 0.5 \\
\hline Left main & 5 & 6 & 5.5 \\
\hline LAD proximal & 3.5 & 3.5 & 3.5 \\
\hline LAD mid & 2.5 & 2.5 & 2.5 \\
\hline LAD distal & 1 & 1 & 1 \\
\hline 1st diagonal & 1 & 1 & 1 \\
\hline 2nd diagonal & 0.5 & 0.5 & 0.5 \\
\hline LCx proximal & 1.5 & 2.5 & 2.0 \\
\hline 1st obtuse marginal & 1 & 1 & 1 \\
\hline LCx distal & 0.5 & 1.5 & 1 \\
\hline 2nd obtuse marginal & 1 & 1 & 1 \\
\hline PDA from LCA & na & 1 & na \\
\hline $\begin{array}{l}\text { PL branch from } \\
\text { LCA }\end{array}$ & na & 0.5 & 0.5 \\
\hline $\begin{array}{l}\text { PL branch } \\
\text { from RCA }\end{array}$ & 0.5 & na & na \\
\hline Intermediate branch & 1 & 1 & 1 \\
\hline \multicolumn{4}{|l|}{ Stenosis severity } \\
\hline Obstructive CAD & 1 & & \\
\hline Nonobstructive CAD & 0.615 & & \\
\hline \multicolumn{4}{|l|}{ Plaque composition } \\
\hline Non-calcified or mixed & 1.5 & & \\
\hline Calcified & 1 & & \\
\hline
\end{tabular}

$R C A$ right coronary artery, $P D A$ posterior descending artery, $L A D$ left anterior descending, $L C x$ left circumflex, $P L$ postero-lateral, $C A D$ coronary artery disease
The CT-LeSc on a patient level was calculated as the sum of the partial CT-LeSc of all evaluable coronary segments. Two cases examples are shown in Fig. 2.

\section{Statistical analysis}

Continuous variables are presented as mean \pm SD or medians (interquartile range) and categorical variables as frequencies with percentages.

The non-parametric Mann-Whitney or Kruskal-Wallis tests were used to compare continuous variables, and the Chi square test to evaluate differences in frequencies. Differences were regarded significant when $p<0.05$ (two-tailed).

Since there are no previous validated cut-offs for the presently described CCTA score, the population with CAD was divided in terciles. A high CT-LeSc was defined with the cut-off for the 3rd tercile (a score $\geq 8.3, \mathrm{n}=116$; $34.8 \%$ of the CAD population) and patients in this group were compared with the remaining population.

Multivariate analyses (binary logistic regression modelenter method) were performed to identify independent predictors of a high CT-LeSc using the demographic and clinical variables presented in Table 2 that had a $p$ value $<0.2$ at univariate analyses. A second multivariable analyses was performed to identify independent predictors among the clinical scores of CAD probability (Diamond-Forrester CAD consortium model and Morise score) and the CV risk score HeartScore.

SPSS version 17.0 (SPSS Inc., Chicago, IL, USA) was used for all statistical analyses.

\section{Results}

In the final study population of 581 patients, 8,136 coronary segments were evaluated. Segments $<2 \mathrm{~mm}(\mathrm{n}=742$; $9.1 \%$ ) or with suboptimal image quality related to artefacts or severe calcification $(\mathrm{n}=120 ; 1.5 \%)$ were excluded.

Most of patients were male (55.8\%) and mean age was $57.6 \pm 11.1$, and $14.6 \%$ were diabetics. This was predominantly a population with low to intermediate CAD probability since $60.1 \%$ had a DF-CAD consortium $<30$ and $87.6 \%$ had a Morise score $<16$. A high cardiovascular risk, as assessed by an HeartScore $\geq 5 \%$, was present in $25.5 \%$ of the patients. In this population, the median calcium score was 1 (IQR 0-93), $23.4 \%$ had a calcium score $(\mathrm{CaSc}) \geq 100$ and $14.3 \%$ had a $\mathrm{CaSc} \geq 75$ th percentile. In the population with CAD, the median CaSc was 64 (IQR 8-200; Table 2).

\section{CT-LeSc}

Overall $(\mathrm{n}=581)$, the median CT-LeSc in this population was 2.2 (IQR 0-6.8). In patients with CAD $(\mathrm{n}=341)$, the 

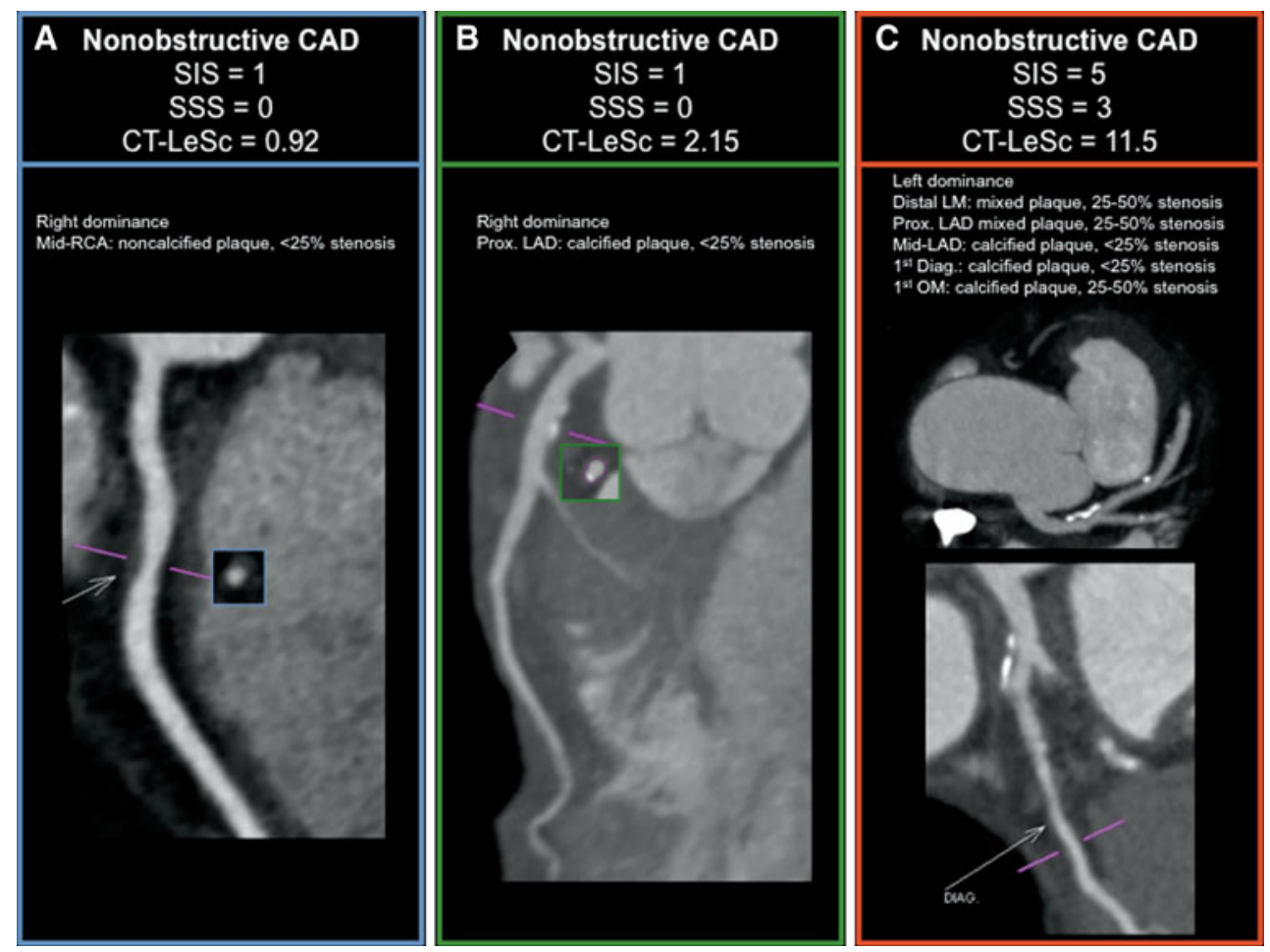

Fig. 2 Three cases examples of patients with nonobstructive CAD stratified by different coronary atherosclerotic burden scores. In panel $A$, a patient with a single lesion in the mid-RCA (weighting for localization $\times$ type of plaque $\times$ stenosis severity $=1 \times 1.5 \times$ $0.615=0.92)$; In panel $B$, a patient with a single proximal LAD lesion $(\mathrm{CT}-\mathrm{LeSc}=3.5 \times 1 \times 0.615=2.15)$. In panel $C$, a patient with left dominance and 5 nonobstructive lesions with a total

median CT-LeSc was 5.8 (IQR 3.2-9.6). Within this population the median CT-LeSc in patients with non-obstructive disease $(\mathrm{n}=263)$ was $4.6(\mathrm{IQR} 2.9-7.7)$ and in patients with obstructive disease $(\mathrm{n}=78)$ it was 11.7 (IQR 8.7-14.4). The terciles in population with CAD were: $T 1$ $\leq 3.7$ (0.3-3.7); $T 2$ (3.8-8.3); T3 $\geq 8.3$ (8.3-24.1).

Regarding the distribution of patients with nonobstructive versus obstructive CAD across the CT-LeSc terciles, most of the patients with nonobstructive CAD were in $\mathrm{T} 1$ $(\mathrm{n}=113,43.0 \%)$ or $\mathrm{T} 2(\mathrm{n}=95,36.1 \%)$, but about one fifth $(\mathrm{n}=55,20.9 \%)$ were in the highest tercile (T3, CTLeSc $\geq 8.3$ ). On the other hand, although most of the patients with obstructive CAD were classified in T3, $21.8 \%$ had a CT-LeSc in lower terciles (T1, $2.6 \%$; T2, $19.2 \%$; Fig. 3).

The median CT-LeSc was significantly higher in males and in the presence of diabetes and hypertension, as well as in patients with a high cardiovascular risk assessed by an HeartScore $\geq 5 \%$. The median CT-LeSc was also significantly higher in patients with a CaSc $\geq 100$ and $\mathrm{CaSc}$ $\geq 75$ th percentile (Fig. 4: Median CT-LeSc in different patient subgroups).

\section{Multivariate predictors}

CT-LeSc $=$ LM $(6 \times 1.5 \times 0.615)+$ prox. $\quad$ LAD $(3.5 \times 1.5 \times$ $0.615)+$ mid-LAD $(2.5 \times 1 \times 0.615)+1$ st Diag. $(1 \times 1 \times 0.615)+$ 1 st $\mathrm{OM}(1 \times 1 \times 0.615)=11.5$. CAD coronary artery disease, CT-LeSc CT Leaman score, SIS segment involvement score, SSS segment stenosis score, $L M$ left main, $L A D$ left anterior descending, $L C x$ left circunflex, $R C A$ right coronary artery, 1 st Diag. first diagonal branch, 1st OM first obtuse marginal branch

In the univariate analysis, a high CT-LeSc was associated with older age ( $\geq 60$ years), diabetes and hypertension. The percentage of male patients and patients with dyslipidemia was also higher in the high CT-LeSc group, but not statistically significant. Patients in the high CT group had also a higher pre-test CAD probability (DF-CAD consortium $\geq 30 \%$ and Morise score $\geq 16$ ) as well as higher CV risk, reflected in the significantly higher percentage of patients with a HeartScore $\geq 5 \%$. Of note, some traditional risk factor as obesity, smoking status and family history of premature CAD were not differently distributed in the two groups, and this was also the case for chest pain (Table 3).

By multivariate analysis, the independent predictors of a high CT-LeSc were: male sex; diabetes, hypertension, Morise score $\geq 16$ and HeartScore $\geq 5$ (Table 4; Fig. 5). Of note, regarding the modifiable risk factors, patients with 
Table 2 Demographic, clinical and CCTA characteristics of the study population

\begin{tabular}{|c|c|}
\hline & All patients $(\mathrm{n}=581)$ \\
\hline \multicolumn{2}{|l|}{ Demographic } \\
\hline Age & $57.6 \pm 11.1$ \\
\hline Male sex & $324(55.8)$ \\
\hline \multicolumn{2}{|l|}{ Risk factors } \\
\hline Obesity (BMI $\geq 30$ ) & $109(18.8)$ \\
\hline Diabetes & 85 (14.6) \\
\hline Hypertension & $364(62.7)$ \\
\hline Dyslipidemia & $360(62.0)$ \\
\hline Smoking & $138(23.8)$ \\
\hline Family history of premature CAD & $194(33.4)$ \\
\hline \multicolumn{2}{|l|}{ Chest pain } \\
\hline Asymptomatic & $270(46.5)$ \\
\hline Noncardiac & $169(29.1)$ \\
\hline Atypical & $109(18.8)$ \\
\hline Typical & $33(5.7)$ \\
\hline \multicolumn{2}{|l|}{ CAD probability } \\
\hline DF-CAD consortium $\geq 70 \%$ & $11(1.9)$ \\
\hline DF-CAD consortium 30-70 \% & $221(38.0)$ \\
\hline DF-CAD consortium $<30 \%$ & $349(60.1)$ \\
\hline Morise score $\geq 16$ & $72(12.4)$ \\
\hline Morise score 9-15 & $369(63.5)$ \\
\hline Morise score $0-8$ & $140(24.1)$ \\
\hline \multicolumn{2}{|l|}{$\mathrm{CV}$ risk } \\
\hline Heart score $\geq 5 \%$ & $148(25.5)$ \\
\hline \multicolumn{2}{|l|}{ Calcium score } \\
\hline Median & $1(0-93)$ \\
\hline Median in patients with CAD & $64(8-200)$ \\
\hline $\mathrm{CaSc} \geq 100$ & $136(23.4)$ \\
\hline CaSc $\geq 75$ th percentile & $83(14.3)$ \\
\hline \multicolumn{2}{|l|}{ CCTA } \\
\hline Normal/no plaque & $240(41.3)$ \\
\hline Nonobstructive CAD & $263(45.3)$ \\
\hline Obstructive CAD & 78 (13.4) \\
\hline \multicolumn{2}{|l|}{ Technical data } \\
\hline Heart rate $(\mathrm{bpm})$ & $65.6 \pm 10.6$ \\
\hline Contrast dose (ml) & $98.9 \pm 14.4$ \\
\hline Radiation dose (mSv) & $4.6 \pm 3.7$ \\
\hline
\end{tabular}

Values are mean $\pm \mathrm{SD}$, median (IQR) or $\mathrm{n}(\%)$

$C A D$ coronary artery disease, $B M I$ body mass index, $D F-C A D$ consortium Diamond-Forrester CAD consortium model, $C V$ cardiovascular, CCTA coronary computed tomography angiography, $\mathrm{CaSc}$ calcium score, $b p m$ beats per minute, $m S v$ milisievert

diabetes had a threefold and patients with hypertension a 2.5-fold higher probability of having a high CT-LeSc.

A high HeartScore $(\geq 5 \%)$ and a high Morise score $(\geq 16)$ were associated respectively with a 2.5 and twofold higher probability of having a high coronary atherosclerotic burden, as assessed by the CT-LeSc.

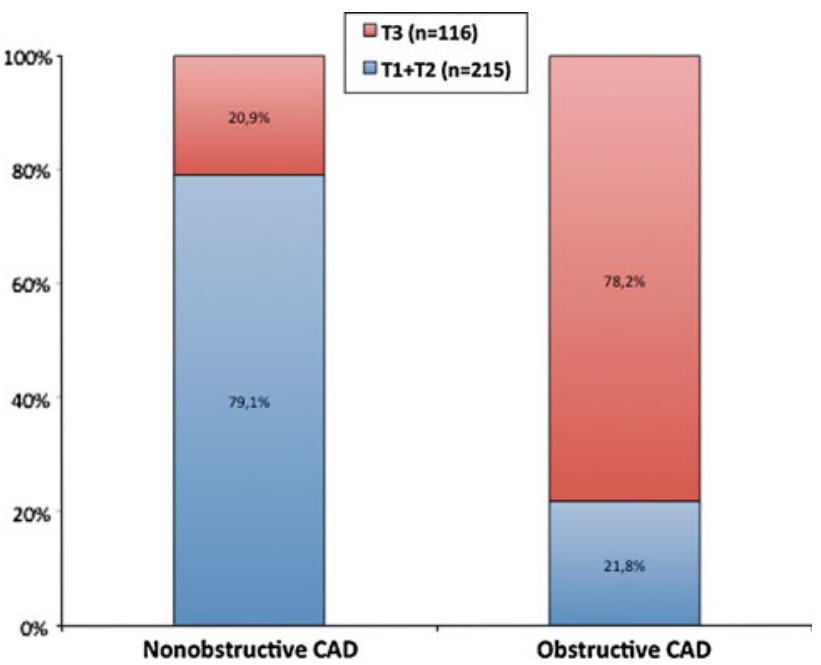

Fig. 3 Distribution of the two subgroups of patients (nonobstructive and obstructive CAD), according to CT-LeSc terciles (T1 + T2 vs T3). $C A D$ coronary artery disease, $T 11$ st tercile, $T 2$ nd tercile, $T 3$ 3rd tercile

\section{Discussion}

The main findings of this study are: (1) Calculation of a cardiac CT adapted Leaman score as a tool to quantify total (obstructive and nonobstructive) coronary atherosclerotic burden, reflecting the comprehensive information about localization, degree of stenosis and type of plaque provided by CCTA is feasible; (2) There was a significant association between the CT-LeSc and diabetes, a well recognized subset of advanced coronary atherosclerotic burden. A high $\mathrm{CV}$ risk (HeartScore) and a high CAD probability (Morise score) were also both associated with nearly a 2-2.5 fold higher probability of having a high coronary atherosclerotic burden, as assessed by the CT-LeSc.

Although the exclusion of obstructive CAD remains presently the main indication to refer a patient for CCTA, this noninvasive diagnostic tool can also provide information regarding the presence of nonobstructive plaques, detecting $\mathrm{CAD}$ at earlier disease stages. Although on a per lesion basis, vulnerability is positively associated with the degree of stenosis, on a per patient level most of the acute events come from nonobstructive lesions [18-20]. It is also recognized that many of the nonstenotic lesions can have a high plaque burden, underestimated by luminal angiograms, since they undergo expansive or positive outward enlargement, and such remodeling is a potential surrogate marker of plaque vulnerability [21]. In the multicenter virtual histology intravascular ultrasound (VH-IVUS) PROSPECT study [22], a large plaque burden, a small lumen area and the presence of a thin cap fibroatheroma were independent predictors of future nonculprit lesion major adverse cardiac events (MACE). In this study, lesions that led to MACE had a high plaque burden by 


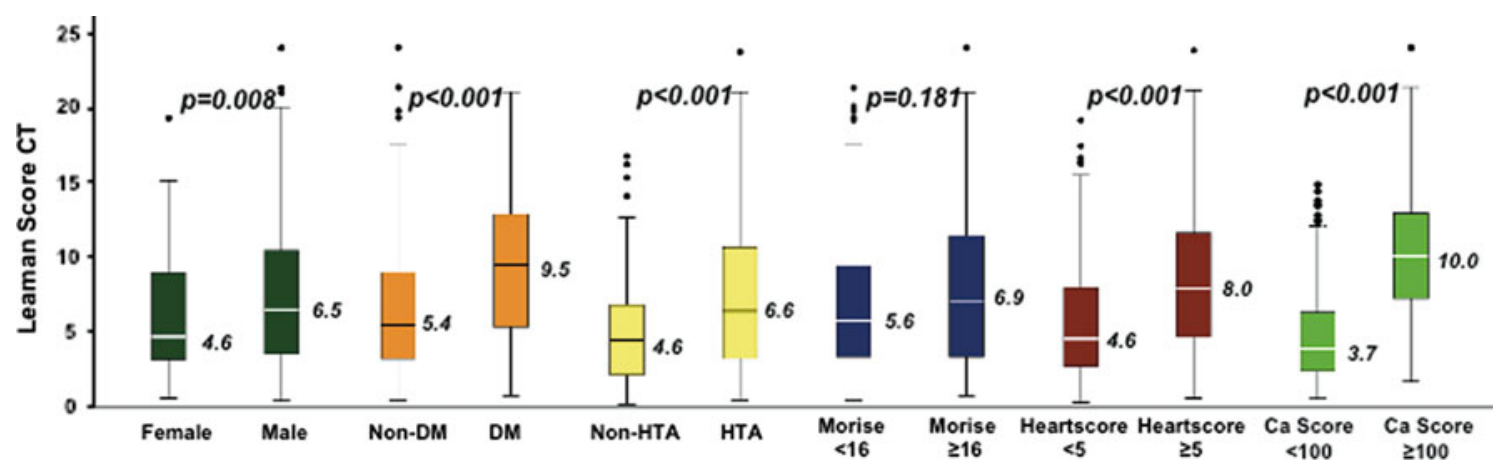

Fig. 4 Median CT-LeSc in different patient subgroups. DM diabetes mellitus, HTA hypertension, CA score calcium score, CT-LeSc CT Leaman score

Table 3 Univariate analysis

\begin{tabular}{lrlr}
\hline & $\begin{array}{l}\text { CT LeSc } \\
\text { T1 }+2 \\
(<8.3)\end{array}$ & $\begin{array}{l}\text { CT } \\
\text { LeSc } \geq\end{array}$ & $p$ \\
& \multicolumn{1}{c}{ T3 $\geq 8.3)$} & \\
\hline Age $\geq 60$ & $126(56.0)$ & $79(68.1)$ & 0.031 \\
Male sex & $138(61.3)$ & $81(69.8)$ & 0.121 \\
BMI $\geq 30$ & $41(18.3)$ & $25(22.1)$ & 0.404 \\
Diabetes & $26(11.6)$ & $36(31.0)$ & $<0.001$ \\
Hypertension & $144(64.0)$ & $98(84.5)$ & $<0.001$ \\
Dyslipidemia & $146(64.9)$ & $87(75.0)$ & 0.057 \\
Smoking & $53(23.6)$ & $31(26.7)$ & 0.520 \\
Family history of premature & $77(34.2)$ & $37(31.9)$ & 0.666 \\
CAD & & & \\
Chest pain & $106(47.3)$ & $59(51.3)$ & 0.487 \\
DF-CAD consortium $\geq 30 \%$ & $66(29.3)$ & $64(55.2)$ & $<0.001$ \\
Morise score $\geq 16$ & $105(46.7)$ & $78(67.2)$ & $<0.001$ \\
Heart score $\geq 5$ & $29(12.9)$ & $24(20.7)$ & 0.060 \\
\hline Valyes & &
\end{tabular}

Values are n $(\%)$; $C A D$ coronary artery disease, $B M I$ body mass index, $D F-C A D$ consortium-diamond-Forrester CAD consortium model

IVUS, but were mild by baseline angiography (mean diameter stenosis $32 \%$ ). The prognostic value of nonobstructive $\mathrm{CAD}$ has also been recently reinforced from large cardiac CT registries (CONFIRM) and meta-analysis [23].

In the large international multicenter CONFIRM registry, all-cause mortality was significantly higher for patients with nonobstructive $\mathrm{CAD}$, as compared with patients without coronary atherosclerosis. One notable finding in this registry is the superimposed survival curves of nonobstructive and 1 vessel obstructive CAD, reinforcing the prognostic impact of nonobstructive coronary lesions [6].

Why a plaque burden CT score?

The main reason is because CAD represents a very heterogeneous condition and there is a need to structure the quantification of the plaque burden and to integrate the
Table 4 Multivariate analysis-independent predictors of a high CTLeSc (3rd tercile, score $\geq 8.3$ )

\begin{tabular}{lrrr}
\hline \multicolumn{1}{c}{ OR } & $(95 \%$ CI $)$ & \multicolumn{1}{c}{$p$} \\
\hline Demographic and clinical variables & & \\
Age $\geq 60$ & 1.370 & $0.819-2.291$ & 0.230 \\
Male sex & 1.732 & $1.035-2.901$ & 0.037 \\
Diabetes & 2.905 & $1.612-5.234$ & $<0.001$ \\
Hypertension & 2.543 & $1.395-4.634$ & 0.002 \\
Dyslipidemia & 1.563 & $0.919-2.660$ & 0.099 \\
Clinical scores & & & \\
Heart score $\geq 5$ & 2.416 & $1.411-4.135$ & 0.001 \\
DF-CAD consortium $\geq 30 \%$ & 1.590 & $0.918-2.754$ & 0.098 \\
Morise $\geq 16$ & 1.971 & $1.060-3.666$ & 0.032 \\
\hline
\end{tabular}

OR odds ratio, DF-CAD consortium Diamond-Forrester CAD consortium model

most important information collected by $\mathrm{CT}$ and finally to homogenize the reporting of CT findings.

There are already some CT scores developed and prognostically validated namely the segment involvement score (SIS) and the segment stenosis score (SSS), but they only reflect some aspects of the coronary atherosclerotic burden, the former only takes into account the number of segments with plaque and the latter the degree of stenosis [6]. The CT-LeSc reflects some of the aspects that are partially included in the SIS (number of segments with plaque) and the SSS (degree of stenosis), and combines these two aspects, and also the localization, on a more comprehensive score.

Why these three components?

Individually, localization of the plaque within the coronary tree, the type of plaques and degree of stenosis are strong predictors of future coronary events.

Since CCTA is able to reliably collect information on these three aspects, a comprehensive score should be able to integrate these components. 
Fig. 5 Independent predictors of a high CT-LeSc (score $\geq 8.3$ ). CT-LeSc CT Leaman score

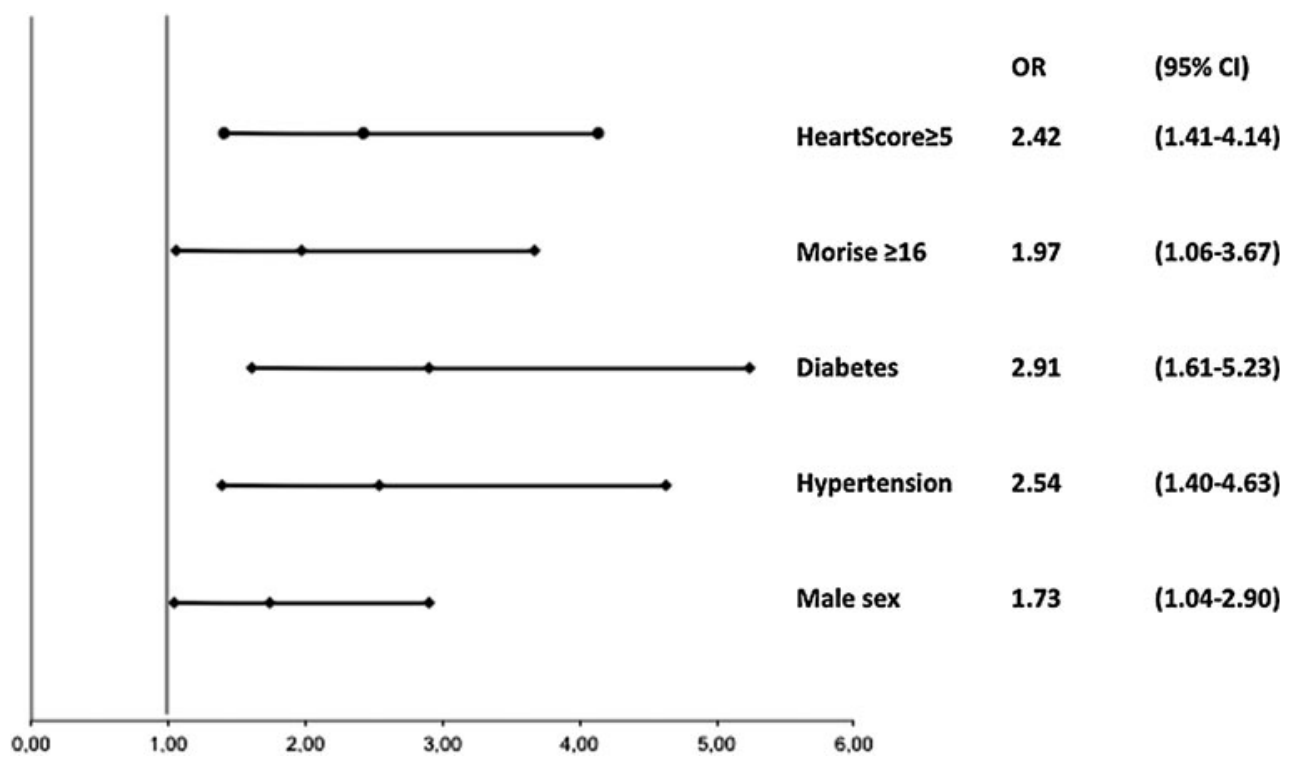

Regarding localization, the original Leaman score was developed as a score to quantify obstructive coronary disease identified with invasive angiography [15]. In this score, with the rational of relative blood supply to the left ventricle, all coronary segments were given a weighting factor, reflecting the relative contribution of blood flow to the left ventricle of each vessel segment, taking also in account the specific right or left dominance systems. Recently, this score was used as the segment weighting factor for the development of the syntax score [24] which has been proven to have a strong prognostic value in different clinical scenarios [25, 26]. In our score, values were also provided for balanced dominance, reflecting more adequately the anatomical variants of the coronary tree.

Plaque composition has been found in both pathological and clinical studies associated with cardiac events [22, 27]. CCTA has shown to be able to characterize plaque composition [28]. Thin cap fibroatheroma is the most common pathological substrate of ACS and in CCTA these plaques appear as noncalcified or mixed plaques [16]. In a recent study by Maurovich-Horvat et al. [16], the frequency of a napkin-ting sign, a CCTA feature of advanced lesions by histology, was similar between noncalcified and mixed plaques, which also reinforces our weighting factor in the CT-LeSc for plaque composition that was the same between these two types (a factor of 1.5), and different from predominantly calcified plaques (a factor of 1).

Regarding the degree of stenosis, we assumed in our scoring system a factor reflecting the proportion of the hazard ratios for obstructive versus nonobstructive observed in the recently large scale CONFIRM registry. By gathering all the nonobstructive $(<25,25-49 \%)$ and obstructive
$(50-69,70-99,100 \%)$ in the same risk categories, this scoring system is expected to have a good intra and interobserver correlation, since the other two weighting factors (localization and plaque calcification) have also an excellent reproducibility and are usually described in CCTA reporting.

\section{Clinical implications}

Many tools are already available to help stratifying patients at risk of a CV event and some scores have been already developed gathering the information provided by the different traditional risk factors, like the Framingham score or the HeartScore. Notwithstanding these observations, accurate prediction of major coronary events on the individual patient level, as opposed to population based studies, remains challenging.

The clinical implications of a score that reflect the extent of coronary atherosclerotic burden is related to the fact that this way we can have a tool to quantify and compare this burden, which is particularly useful when reporting a CCTA of a patient without obstructive CAD, but in whom the extent of nonobstructive CAD could lead to a reclassification of his risk profile and thereby his cardiovascular treatment.

Of note in our study is the fact that although the CTLeSc, by having the degree of stenosis in its composition, tends to favour patients with obstructive CAD, we were able to demonstrate that a significant percentage (20.9\%) of patients with nonobstructive CAD had in fact a CT-LeSc in the highest tercile (T3). Conversely, among patients with obstructive CAD, about one fifth had a coronary 
atherosclerotic burden, as assessed by the CT-LeSc, in lower terciles.

The CT-LeSc, by having a weight related to the localization, it reflects not only the extent of CAD but also the expected clinical consequences in case that the more proximal lesions evolve to a significant stenosis or become unstable and trigger a coronary event.

In our study, a high HeartScore and a high Morise score were both associated with nearly a 2-2.5 fold higher probability of having a high coronary atherosclerotic burden, as assessed by the CT-LeSc. This could be expected for the HeartScore, as it was develop as a tool to predict cardiovascular risk. In the case of the Morise score, is was developed and validated as a clinical tool to estimate the probability of CAD, but it has also been linked to cardiovascular outcomes [29]. The Diamond-Forrester was not an independent predictor of a high CT-LeSc and although we used the recently calibrated CAD consortium model [12], is has been developed and calibrated for obstructive CAD identified with invasive angiography and doesn't take in account the cardiovascular risk factors in its composition.

\section{Limitations}

There are a number of limitations related to this report: (1) This is a single center data with medium size cohort; (2) High prevalence of low CAD probability/CV risk patients. The population included in our study was mainly composed of patients with low to intermediate CAD probability and CV risk. Nevertheless, CAD was present in nearly $60 \%$ of the patients and this reflects the daily practice and the recommendations that high CAD probability patients have not an appropriate indication for CCTA [30]; (3) For the weighting factor of plaque composition, we used a multiplication factor of 1.5 for mixed and noncalcified plaques. Although this was an arbitrary factor, this is in line with several CCTA prognostic studies that demonstrated lower hazard ratios for calcified plaques and reflects an assumption of less plaque vulnerability of calcified plaques. (4) Lack of prognostic validation: the aim of this study was to describe a CCTA score to quantify total coronary atherosclerotic burden and to identify its clinical predictors. Future studies will be needed to provide a prognostic validation of this described CT-LeSc.

\section{Conclusions}

The calculation of the CCTA-adapted Leaman score as a tool to quantify total (obstructive and nonobstructive) coronary atherosclerotic burden, reflecting the comprehensive information about localization, degree of stenosis and type of plaque provided by CCTA is feasible. There was a significant association between the CT-LeSc and some traditional demographic and clinical risk factors. In face of this association, we expect this score to be a useful tool to quantify the coronary atherosclerotic burden evaluated by CCTA and it is expected to convey prognostic information, and this should be evaluated in future studies.

About one fifth of the patients with nonobstructive CAD had a CT-LeSc in the highest tercile, which could potentially lead to a reclassification of the risk profile of these subset of patients identified by CCTA, once the prognostic value of the CT-LeSc is validated.

Conflict of interest There were no sources of funding and the authors have no conflicts of interest related to this manuscript.

\section{References}

1. Lloyd-Jones D, Adams R, Carnethon M, De Simone G, Ferguson TB, Flegal K et al (2009) Heart disease and stroke statistics-2009 update: a report from the American Heart Association Statistics Committee and Stroke Statistics Subcommittee. Circulation 119(3):480-486

2. Braunwald E (2006) Epilogue: what do clinicians expect from imagers? J Am Coll Cardiol 47(8 Suppl):C101-C103

3. Meijboom WB, van Mieghem CA, Mollet NR, Pugliese F, Weustink AC, van Pelt $\mathrm{N}$ et al (2007) 64-slice computed tomography coronary angiography in patients with high, intermediate, or low pretest probability of significant coronary artery disease. J Am Coll Cardiol 50(15):1469-1475

4. Weustink AC, Mollet NR, Neefjes LA, van Straten M, Neoh E, Kyrzopoulos S et al (2009) Preserved diagnostic performance of dual-source CT coronary angiography with reduced radiation exposure and cancer risk. Radiology 252(1):53-60

5. Chow BJ, Small G, Yam Y, Chen L, Achenbach S, Al-Mallah M et al (2011) Incremental prognostic value of cardiac computed tomography in coronary artery disease using CONFIRM: COroNary computed tomography angiography evaluation for clinical outcomes: an InteRnational Multicenter registry. Circ Cardiovasc Imaging 4(5):463-472

6. Min JK, Shaw LJ, Devereux RB, Okin PM, Weinsaft JW, Russo DJ et al (2007) Prognostic value of multidetector coronary computed tomographic angiography for prediction of all-cause mortality. J Am Coll Cardiol 50(12):1161-1170

7. Perk J, De Backer G, Gohlke H, Graham I, Reiner Z, Verschuren $M$ et al (2012) European Guidelines on cardiovascular disease prevention in clinical practice (version 2012): the Fifth Joint Task Force of the European Society of Cardiology and Other Societies on Cardiovascular Disease Prevention in Clinical Practice (constituted by representatives of nine societies and by invited experts) * Developed with the special contribution of the European Association for Cardiovascular Prevention \& Rehabilitation (EACPR). Eur Heart J 33(13):1635-1701

8. Report of the Expert Committee on the Diagnosis and Classification of Diabetes Mellitus (1997) Diabetes Care 20(7):1183-1197

9. Expert Panel on Detection E, Treatment of High Blood Cholesterol in A (2001) Executive summary of the third report of the National Cholesterol Education Program (NCEP) expert panel on detection, evaluation, and treatment of high blood cholesterol in adults (adult treatment panel III). JAMA 285(19):2486-2497

10. European Society of Hypertension-European Society of Cardiology Guidelines C (2003) 2003 European Society of HypertensionEuropean Society of Cardiology guidelines for the management of arterial hypertension. J Hypertens 21(6):1011-1053 
11. Taylor AJ, Bindeman J, Feuerstein I, Cao F, Brazaitis M, O'Malley PG (2005) Coronary calcium independently predicts incident premature coronary heart disease over measured cardiovascular risk factors: mean three-year outcomes in the Prospective Army Coronary Calcium (PACC) project. J Am Coll Cardiol 46(5):807-814

12. Genders TS, Steyerberg EW, Alkadhi H, Leschka S, Desbiolles L, Nieman K et al (2011) A clinical prediction rule for the diagnosis of coronary artery disease: validation, updating, and extension. Eur Heart J 32(11):1316-1330

13. Morise AP, Haddad WJ, Beckner D (1997) Development and validation of a clinical score to estimate the probability of coronary artery disease in men and women presenting with suspected coronary disease. Am J Med 102(4):350-356

14. Raff GL, Abidov A, Achenbach S, Berman DS, Boxt LM, Budoff MJ et al (2009) SCCT guidelines for the interpretation and reporting of coronary computed tomographic angiography. J Cardiovasc Comput Tomogr 3(2):122-136

15. Leaman DM, Brower RW, Meester GT, Serruys P, van den Brand M (1981) Coronary artery atherosclerosis: severity of the disease, severity of angina pectoris and compromised left ventricular function. Circulation 63(2):285-299

16. Maurovich-Horvat P, Schlett CL, Alkadhi H, Nakano M, Otsuka F, Stolzmann P et al (2012) The napkin-ring sign indicates advanced atherosclerotic lesions in coronary CT angiography. JACC Cardiovasc Imaging 5(12):1243-1252

17. Andreini D, Pontone G, Mushtaq S, Bartorelli AL, Bertella E, Antonioli L et al (2012) A long-term prognostic value of coronary CT angiography in suspected coronary artery disease. JACC Cardiovasc Imaging 5(7):690-701

18. Little WC, Downes TR, Applegate RJ (1991) The underlying coronary lesion in myocardial infarction: implications for coronary angiography. Clin Cardiol 14(11):868-874

19. Falk E, Shah PK, Fuster V (1995) Coronary plaque disruption. Circulation 92(3):657-671

20. Libby P, Theroux P (2005) Pathophysiology of coronary artery disease. Circulation 111(25):3481-3488

21. Naghavi M, Libby P, Falk E, Casscells SW, Litovsky S, Rumberger $\mathbf{J}$ et al (2003) From vulnerable plaque to vulnerable patient: a call for new definitions and risk assessment strategies: part I. Circulation 108(14):1664-1672

22. Stone GW, Maehara A, Lansky AJ, de Bruyne B, Cristea E, Mintz GS et al (2011) A prospective natural-history study of coronary atherosclerosis. N Engl J Med 364(3):226-235
23. Bamberg F, Sommer WH, Hoffmann V, Achenbach S, Nikolaou $\mathrm{K}$, Conen D et al (2011) Meta-analysis and systematic review of the long-term predictive value of assessment of coronary atherosclerosis by contrast-enhanced coronary computed tomography angiography. J Am Coll Cardiol 57(24):2426-2436

24. Sianos G, Morel MA, Kappetein AP, Morice MC, Colombo A, Dawkins K et al (2005) The SYNTAX score: an angiographic tool grading the complexity of coronary artery disease. EuroIntervention 1(2):219-227

25. Serruys PW, Morice MC, Kappetein AP, Colombo A, Holmes DR, Mack MJ et al (2009) Percutaneous coronary intervention versus coronary-artery bypass grafting for severe coronary artery disease. N Engl J Med 360(10):961-972

26. Brito J, Teles R, Almeida M, de Araujo Goncalves P, Raposo L, Sousa $\mathrm{P}$ et al (2011) Predictive value of SYNTAX score in risk stratification of patients undergoing unprotected left main coronary artery angioplasty. J Invasive Cardiol 23(12):494-499

27. Virmani R, Kolodgie FD, Burke AP, Farb A, Schwartz SM (2000) Lessons from sudden coronary death: a comprehensive morphological classification scheme for atherosclerotic lesions. Arterioscler Thromb Vasc Biol 20(5):1262-1275

28. Pundziute G, Schuijf JD, Jukema JW, Decramer I, Sarno G, Vanhoenacker PK et al (2008) Head-to-head comparison of coronary plaque evaluation between multislice computed tomography and intravascular ultrasound radiofrequency data analysis. JACC Cardiovasc Interv 1(2):176-182

29. Morise AP, Jalisi F (2003) Evaluation of pretest and exercise test scores to assess all-cause mortality in unselected patients presenting for exercise testing with symptoms of suspected coronary artery disease. J Am Coll Cardiol 42(5):842-850

30. Taylor AJ, Cerqueira M, Hodgson JM, Mark D, Min J, O'Gara P et al (2010) ACCF/SCCT/ACR/AHA/ASE/ASNC/NASCI/SCAI/ SCMR 2010 appropriate use criteria for cardiac computed tomography. A report of the American College of Cardiology Foundation Appropriate Use Criteria Task Force, the Society of Cardiovascular Computed Tomography, the American College of Radiology, the American Heart Association, the American Society of Echocardiography, the American Society of Nuclear Cardiology, the North American Society for Cardiovascular Imaging, the Society for Cardiovascular Angiography and Interventions, and the Society for Cardiovascular Magnetic Resonance. J Am Coll Cardiol 56(22):1864-1894 